\title{
HOST-RANGE EVOLUTION: ADAPTATION AND TRADE-OFFS IN FITNESS OF MITES ON ALTERNATIVE HOSTS
}

\author{
Anurag A. Agrawal ${ }^{1}$ \\ Center for Population Biology, University of California, Davis, California 95616 USA
}

\begin{abstract}
Trade-offs in fitness on different host plants has been a central hypothesis in explaining the evolutionary specialization of herbivores. Surprisingly, only a few studies have documented such trade-offs. In this paper, I present results from a selection experiment that demonstrates trade-offs in host plant use for a polyphagous spider mite. Although adaptation to a novel poor-quality host did not result in detectable costs on a favorable host, spider mites that had adapted to a poor-quality host lost their ability to tolerate the poor-quality host when they were reverted to the favorable host for several generations. Trade-offs in fitness on alternative hosts among herbivorous spider mites remains one of the classic empirical examples of constraints on the evolution of host range.

Adaptation to the novel poor-quality host was not associated with adaptation to a related host-plant species or to particular host-plant chemicals that I assayed. Thus, the complexity of host-plant defenses may restrict host shifts to single species of novel host plants, and adaptive zone shifts onto entire groups of plants predicted by the Ehrlich and Raven Model may be rare. Spider mite performance was genetically associated with host-plant preference. Mites from the control population showed a significant preference for the favorable host plant, whereas mites adapted to the novel host plant showed no preference. Finally, although induced plant responses to herbivory in the poor-quality host decreased the fitness of unselected mites, induced responses resulted in higher fitness of adapted mites. These results suggest that spider mites that rapidly adapt to particular host plants can overcome constitutive and inducible plant defenses.

Key words: cotton; cucumber; Cucumis sativus; cucurbitacins, reduction of mite fitness; Gossypium hirsutum; herbivory; host-range evolution and specialization; induced plant resistance; induced resistance, spider mites; plant-insect interactions; spider mites; Tetranychus urticae.
\end{abstract}

\section{INTRODUCTION}

Theory predicts that polyphagy in herbivorous arthropods is restricted by trade-offs in performance on different host plants (Futuyma and Moreno 1988, Jaenike 1990, Thompson 1994). Most herbivorous arthropods are restricted to feeding on relatively few plant families, and it is believed that this host-range limitation may be due to trade-offs in fitness on alternative hosts (Fox and Morrow 1981). Trade-offs in fitness may be due to differences in adaptation to plant defenses such as chemical detoxification ability. Surprisingly, evidence for such trade-offs has been found in only a few studies (Gould 1979, Karban 1989, Fry 1990, Karowe 1990, Via 1991, MacKenzie 1996) and such tradeoffs have more often not been found (Bernays and Graham 1988, Thompson 1996, Abrahamson and Weis 1997, and references therein). In cases where tradeoffs have been found, the mechanisms causing the trade-offs are unknown. Trade-offs in host-plant use driven by plant chemistry have been argued to play a

Received 20 October 1997; revised 7 October 1998; accepted 23 November 1998; final version received 11 January 1999.

${ }^{1}$ Address for correspondence: Department of Botany, University of Toronto, 25 Wilcocks Avenue, Toronto, Ontario, Canada M5S 3B2. E-mail: agrawal@botany.utoronto.ca central role in herbivore specialization (Schultz 1988). In some cases, adapted (or specialized) herbivores rely on secondary compounds as host-finding cues (Giamoustaris and Mithen 1995), sequestered defenses (Bowers 1993), or even as a source of energy (Rosenthal et al. 1978), thereby potentially reducing a herbivore's ability to maximize fitness on alternative hosts. In other cases, adapted herbivores may be susceptible to the negative effects of plant defenses even though they prefer those plants (Adler et al. 1995), and factors other than chemistry may be more important in specialization (Janzen 1985, Bernays and Graham 1988, Joshi and Thompson 1995, Fry 1996, Björkman et al. 1997).

Two important components in host-range ecology and evolution are host preference and subsequent performance on the plant. Although it has long been suggested that host-plant preference and performance should be positively correlated, the theory and evidence have not been well matched (Mayhew 1997). Many studies have found no correlation or even a negative association between herbivore preference and performance (Thompson 1988, Courtney and Kibota 1990, Abrahamson and Weis 1997, Mayhew 1997, but see Price 1994). Others have found that some host plants that appear to be of poor food quality in isolation are 
FIG. 1. Adult female spider mites and a mite egg on a cotton leaf. The mite egg has a diameter of $\sim 0.6 \mathrm{~mm}$. Photo courtesy of Jack Kelly Clark and the University of California Statewide IPM Project.

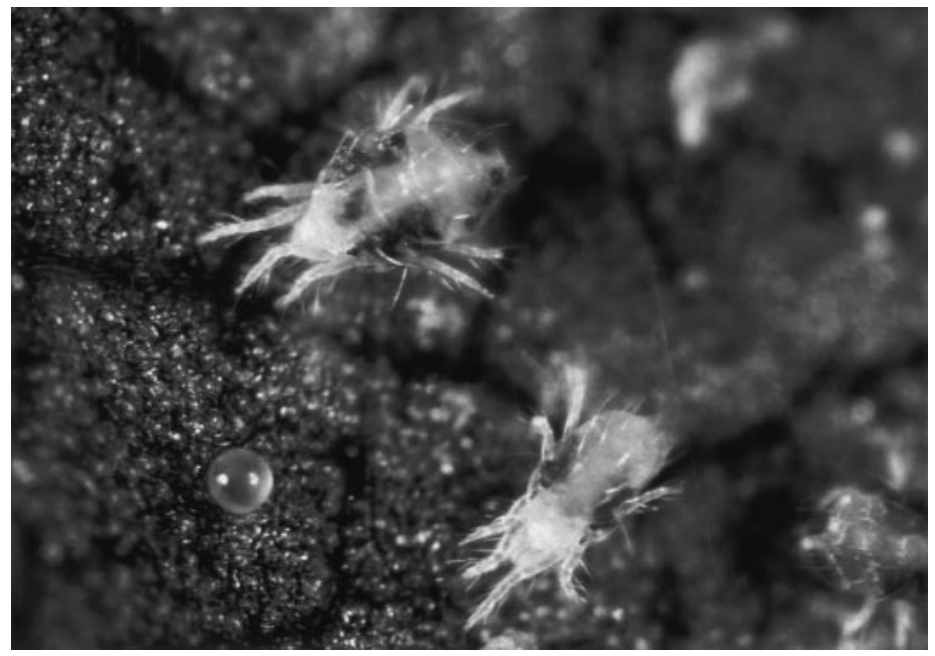

"protective" in the ecological context of predators and competitors. For example, Björkman et al. (1997) found that pine sawflies preferred to oviposit on trees that had a high content of resins. In the absence of predators, the larvae on high-resin trees performed poorly relative to larvae on low-resin trees; however, uncaged larvae on high-resin trees suffered greatly reduced parasitism. Although ecological studies of phenotypic associations between preference and performance provide insight into the potential constraints and selection pressures on the evolution of host range, selection experiments provide a unique tool to study the genetic basis and linkage of these traits. However, few studies have selected for high herbivore performance to particular host plants and examined the correlated response in preference (Fry 1989, Gotoh et al. 1993).

Here I replicate and extend Gould's (1979) classic experiments on trade-offs in host use of the polyphagous two-spotted spider mite, Tetranychus urticae. After demonstrating that there is a trade-off between performance of the spider mites on two host plants, I consider potential mechanisms of adaptation to the host plant. I then examine whether adapted herbivores are susceptible to induced plant defenses and whether adaptation to a poor-quality novel host plant correlated with changes in host-plant preference. Specifically I asked: (1) Can a field-collected population of spider mites adapt to a novel host plant that is usually of poor quality (cucumber)? (2) Is there a cost associated with adaptation to this poor-quality host plant when subsequently feeding on a favorable host? (3) Does the population of mites that is adapted to the poor-quality host plant lose its higher fitness on the poor-quality host plant after it is reverted for several generations to feeding on the favorable host? (4) Is adaptation to the host plant associated with the ability to tolerate particular chemicals (cucurbitacins)? (5) Is adaptation to the poor-quality host plant correlated with high performance on a closely related novel host plant? (6) Is adaptation to the host plant correlated with a change in host-plant preference of the spider mites? And finally, (7) Are spider mites that are adapted to a particular host plant susceptible to the negative effects of induced plant resistance in that host plant?

\section{Materials And Methods}

Study system and general procedures

Tetranychus urticae is a widely distributed spider mite species that feeds on crops and wild plants in most areas of the world (Jeppson et al. 1975) (Fig. 1). T. urticae is an extreme generalist with over 900 recorded host plants and often forms genetically differentiated populations with somewhat more narrow host ranges (Gotoh et al. 1993, Navjas 1998). I collected several hundred T. urticae from cotton, bean, roses, and morning glories (Convolvulus arvensis) in Davis, California, USA. These mites were maintained in a laboratory colony on cotton (Gossypium hirsutum var. Acala SJ-2), a favorable host plant. The colony of thousands of mites was maintained on $\sim 350$ cotton seedlings. After 3-4 generations (one month), this colony was split into two colonies: the control line (c-line) and the line selected for adaptation to cucumber (s-line). The c-line was maintained on cotton plants and the s-line was maintained on cucumber plants that constitutively produce cucurbitacins (Cucumis sativus var. Marketmore 76). After the s-line mites had reproduced on cucumber for eight generations, a reversion line (r-line) was started with several thousand mites from the s-line, which were subsequently maintained on cotton plants (identical to the c-line).

\section{Host-plant chemistry}

Cucumber plants and related species in the Cucurbitaceae contain cucurbitacins. Cucurbitacins are oxygenated tetracyclic triterpenes that have been shown to have emetic, antineoplastic, and cytotoxic effects on 
herbivores, resulting in feeding and oviposition deterrence and reduced growth of consumers (Miro 1995, Tallamy et al. 1997). Cucurbitacins are the most bittertasting compounds known and can be detected by humans in dilutions of one part per billion $\left(10^{9}\right)$ (Metcalf et al. 1980, Metcalf and Lampman 1989). Cucurbitacins have been demonstrated to function defensively against several widespread generalist herbivores, including spider mites, roaches, several beetles, lepidopteran larvae, mice, and vertebrate grazers (Da Costa and Jones 1971, Gould 1978, Metcalf and Lampman 1989, Tallamy et al. 1997). Although it is not known exactly how cucurbitacins negatively affect herbivorous spider mites, it has been found that cucurbitacins reduce mite survivorship and fecundity, but do not affect their host-plant preference (Gould 1978). Cotyledons of the variety of cucumber used in this experiment produce up to $800 \mu \mathrm{g}$ cucurbitacin/g dry mass, while the first true leaves produce up to $100 \mu \mathrm{g} \mathrm{cu}-$ curbitacin/g (Agrawal et al. 1999). Cotton plants also contain suites of putatively defensive secondary compounds ( $\mathrm{Bi}$ et al. 1997). In spite of this, tetranychid spider mites appear to be well adapted to cotton plants and are severe pests of cotton in agricultural fields (Wilson et al. 1987, Agrawal and Karban 1997, Sadras and Wilson 1997).

\section{Adaptation and trade-off assays and general procedures}

Over the course of 8 mo, spider mites from selection and control colonies were assayed for adaptation to cucumber plants three separate times. Two weeks before each assay, several hundred mites were taken from each colony and used to inoculate a pot of 20-30 cotton seedlings. This allowed each of the colonies to complete at least one full generation under identical conditions to minimize potential maternal environmental effects. This procedure was employed in all of the experiments described below. To assay adaptation to the cucumber plants, I made three enclosures $\left(\sim 0.8 \mathrm{~cm}^{2}\right)$ using a thin ring of sticky Tanglefoot (Tanglefoot Company, Grand Rapids, Michigan, USA) on the adaxial side of each intact first true leaf on each potted cucumber plant. Each enclosure was inoculated with three adult female spider mites. Dead adult mites were rarely found in the Tanglefoot or inside of the enclosure, indicating that adult mortality was not a major contributor to mite fitness. All of the assays were replicated with 15-25 plants, each having one enclosure for each of the lines being tested.

Fitness of the mites was measured by counting the total number of progeny left in each enclosure after 1 wk, and the fitness of the lines was compared using ANOVA. Spider mite generation time from egg to egg is $\sim 10 \mathrm{~d}$. Fitness assays were conducted after $\sim 5,13$, and 20 generations (at 50,130, and $200 \mathrm{~d}$ after beginning the experiment). Separate replicates were set up with new plants and mites for each assay at generations
5, 13, and 20. Mite fitness was primarily composed of adult fecundity and juvenile survivorship. There were not problems with contamination or movement of mites between enclosures.

To measure if there were fitness costs associated with feeding on cucumber (novel host) I assayed fitness of the c- and s-lines on favorable cotton plants twice during the experiment. Procedures were identical to the assays on cucumber, except that cotton cotyledons were used as the assay plant.

\section{Adaptation to a related host plant and host-plant chemistry}

These experiments were designed to determine if the s-line spider mites adapting to cucumbers were evolving towards an "adaptive zone." Such an evolutionary shift predicts that mites adapted to cucumbers would also have high performance on related plants in the Cucurbitaceae. I tested if adaptation to cucumber plants affected the fitness of mites feeding on zucchini plants. Experiments were conducted as above to determine fitness of c- and s-line mites on a bitter variety of zucchini, Cucurbita pepo variety Ambassador $(n=20$ plants for each treatment). Bitter zucchini plants contain cucurbitacin-d, a molecule related to cucurbitacin-c found in cucumber plants.

I also used cucurbitacin-d extracted from Cucurbita andreana fruits (Halaweish and Tallamy 1993) to test if the s-line mites could tolerate cucurbitacins better than c-line mites. Cucurbitacin-c was unavailable for this experiment. Tanglefoot enclosures on intact cotton cotyledons were painted with $\sim 0.1 \mathrm{~mL}$ solution of either cucurbitacin-d $(0.5 \mathrm{mg}$ cucurbitacin-d in $1 \mathrm{~mL}$ methanol) or methanol alone (controls). Both c- and s-line mites were assayed as described above, and this experiment was conducted twice ( $n=19-20$ plants each treatment, each trial). The fitness of the lines was compared using ANOVA, with selection line, cucurbitacins, and trial as main effects.

\section{Evolution of spider mite preference}

This experiment was conducted to see whether adaptation to cucumber plants was genetically associated with a change in the preference for host plants (cotton vs. cucumber). Several hundred spider mites from the c-line and the s-line were inoculated on bean (Phaseolus vulgaris var. Bush Lima) plants, a favorable host plant. These mites were left on multiple separate bean plants for $2 \mathrm{wk}$ in order to minimize potential maternal environmental effects that could influence host-plant preference. Cotton seeds and cucumber seeds were then germinated in the same pot so that the emerging cotyledons of these two species would be touching. To test for preference in both the c- and s-line mites, a $1-\mathrm{cm}^{2}$ piece of bean leaf tissue infested with mites was placed on the area where the cotyledons of cotton and cucumber were touching ( $n=20$ pairs for each line). The bean leaf tissue dried up in $\sim 3 \mathrm{~h}$. After $24 \mathrm{~h}$ all 


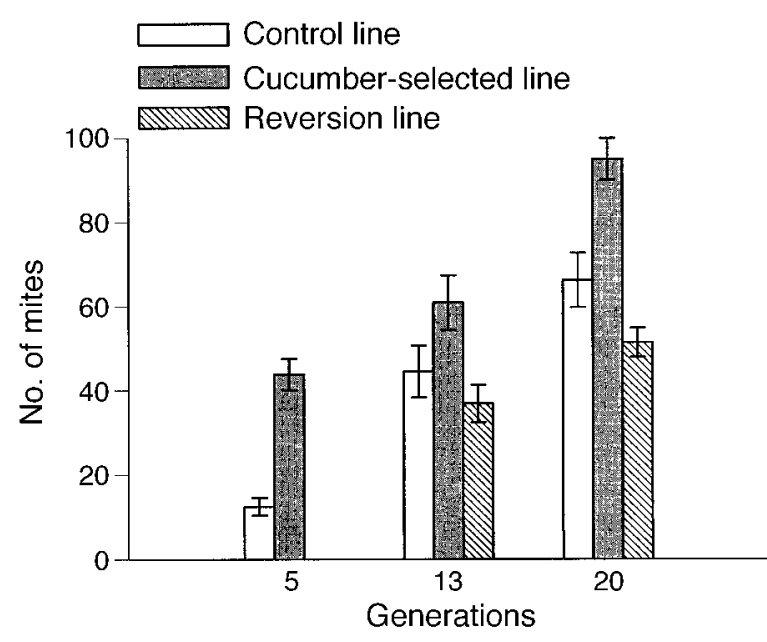

FIG. 2. Fitness of spider mites on the novel host plant (cucumber) on three sampling dates. The control line (c-line) was maintained on cotton, the cucumber-selected line (s-line) was maintained on cucumber plants, and the reversion line (r-line) was switched from cucumber to cotton plants after eight generations. Data are means $\pm 1 \mathrm{SE}$.

mites on the cotton and cucumber cotyledons were counted. A short assay time was chosen to minimize plant-related mortality of mites. Mite preference was estimated by comparing the number of mites on each plant using a paired $t$ test.

\section{Host-plant adaptation and induced plant resistance}

Induced plant resistance to herbivores is a change in the plant that reduces the preference or performance of subsequently attacking herbivores (Agrawal 1998, 1999). Cucumber plants with spider mite herbivory have $30-60 \%$ higher concentrations of cucurbitacins than undamaged plants (Agrawal et al. 1999). In addition, previous mite herbivory induces resistance against subsequent attack. Exposure of cucumber cotyledons to spider mites results in a $40 \%$ decrease in the population growth of mites on the newly formed leaves compared to that on uninduced controls plants (Agrawal et al. 1999). To test if spider mites that are adapted to cucumber plants are less sensitive to the induced responses of cucumber plants, I conducted experiments to examine the effects of induction on c- and $\mathrm{s}$-line mites. The method for the induction process and subsequent challenge follows. I placed 15 adult female spider mites on the newly emerged cucumber cotyledons. The mites were allowed to feed for $3 \mathrm{~d}$, after which all of the plants (including controls) were dipped in dicofol (Kelthane, Rohm and Haas Company, Philadelphia, Pennsylvania, USA), a non-systemic miticide. At this time, the first true leaf was only a bud. Seven to 10 days later, when the first true leaf had fully expanded, it was isolated by placing a thin ring of Tanglefoot around the petiole near the base of the leaf. Each first true leaf was then inoculated with three adult female mites from either the c- or the s-lines. Mite fitness was determined by counting all progeny after 1 wk. This experiment was conducted 2 times $(n=14-$ 19 plants for each treatment, each trial) and was analyzed using an ANOVA with mite selection line, induction treatment, and trial as main effects.

\section{RESULTS}

\section{Mite adaptation and trade-offs on alternative hosts}

After approximately five generations, spider mites showed a response to selection, and mites selected for adaptation to cucumber performed significantly better on cucumber plants than did unselected control mites. These differences were also evident on the subsequent two assays after 13 and 20 generations (Fig. 2, Table $1)$. Mites that were selected for adaptation to poorquality cucumber and then reverted to feeding on favorable cotton plants quickly lost their ability to perform well on cucumber plants and performed significantly worse on cucumber plants than did both controls and mites maintained on cucumber (Fig. 2, Table 1).

A trade-off between adaptation to cucumber and cotton plants was not evident, however, when I tested for fitness costs associated with adaptation to cucumber on favorable cotton plants (Fig. 3, Table 2). On the first assay date the mites adapted to cucumber actually had higher fitness on cotton plants than did controls that had been maintained on cotton (Fig. 3, Table 2). How-

TABLE 1. ANOVA table for mite adaptation to the novel host plant.

\begin{tabular}{lrrrr}
\hline \hline \multicolumn{1}{c}{ Source of variation } & \multicolumn{1}{c}{ df } & \multicolumn{1}{c}{ MS } & \multicolumn{1}{c}{$F$} & \multicolumn{1}{c}{$P$} \\
\hline Selection line (generation 5) & 1 & 8128.125 & 57.682 & $<0.001$ \\
Error & 30 & 140.912 & & \\
Selection line & 2 & 11365.569 & 19.612 & $<0.001$ \\
Generation (13 and 20) & 1 & 15884.629 & 27.411 & $<0.001$ \\
Selection line $\times$ Generation & 2 & 954.159 & 1.646 & 0.197 \\
Error & 110 & 579.509 & & \\
Contrast: c-line vs. s-line & 1110 & 9785.946 & 16.887 & $<0.001$ \\
Contrast: c-line vs. r-line & 1110 & 2465.192 & 4.254 & 0.042 \\
\hline
\end{tabular}

Notes: Fitness of spider mites in the control line (c-line) and the cucumber-adatped line (sline) was compared at five mite generations (Fig. 2); fitness of all three lines (c-, s-, and reversion [r-] line) was assayed at 13 and 20 mite generations. Contrasts are from the second analysis with all of the lines being assayed. 


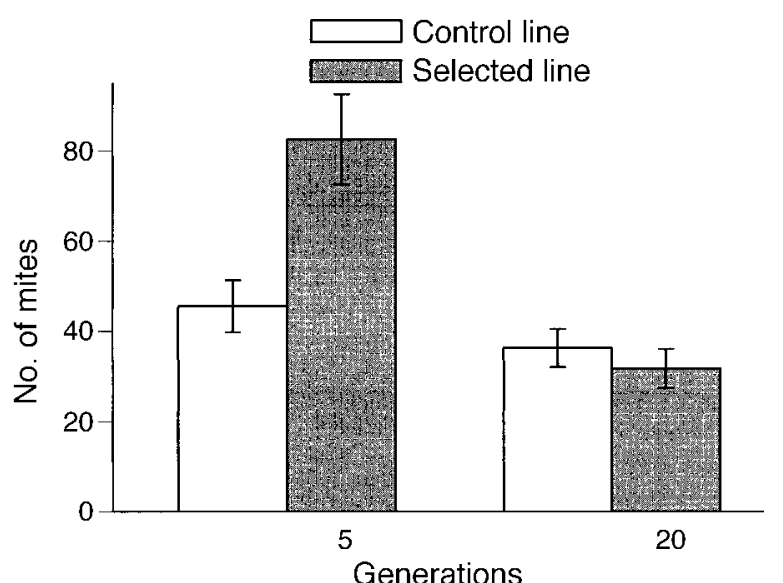

FIG. 3. Fitness of spider mites in the control line (c-line) and the cucumber-adapted line (s-line) on favorable cotton plants on two sampling dates. Data are means \pm 1 SE.

ever, on the second assay date, after $\sim 20$ generations, the fitness of the c-and s-line mites was virtually equal on the cotton plants. The significant line $\times$ generation interaction term indicates this shift in fitness differences over time (Table 2).

\section{Adaptation to a related host plant and host-plant chemistry}

The fitness of mites from both lines was negatively affected by cucurbitacin-d extracted from zucchini, and painted on cotton plants (Fig. 4A, Table 3). Similarly, the c-line and s-line mites had an equally low fitness on zucchini plants, which contain natural cucurbitacin-d (Fig. 4B, $t=-0.629$, df $=19, P=0.537$ ).

\section{Evolution of spider mite preference}

Preference of the mites was significantly affected by selection line (Fig. 5). Mites from the control line, which had been on cotton for 20 generations, showed a significant preference for cotton over cucumber cotyledons (Fig. 5a, $t=4.057$, df $=19, P=0.001$ ). Cucumber-selected mites (s-line) showed no preference for cotton or cucumber plants (Fig. 5b, $t=-0.985$, df $=19, P=0.337)$.

\section{Host-plant adaptation and induced plant resistance}

Induced responses to herbivory triggered by mite feeding on cucumber cotyledons had a negative impact on c-line mite (maintained on cotton) fitness but a pos-

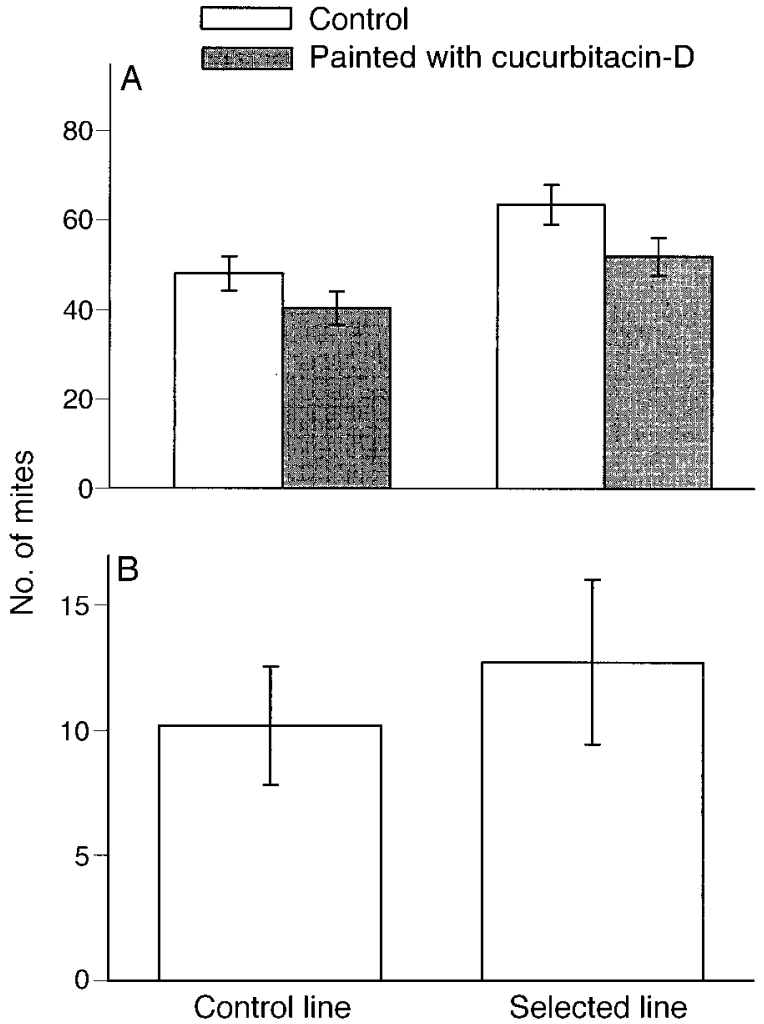

FIG. 4. Fitness of spider mites in the control line (c-line) and the cucumber-adapted line (s-line) (A) on favorable cotton plants and on cotton plants painted with a solution containing cucurbitacin-d and (B) on novel zucchini plants (containing cucurbitacin-d). Data are means $\pm 1 \mathrm{SE}$.

itive effect on s-line (maintained on cucumber) mite fitness in both trials (Fig. 6, Table 4). The main effect of induction was masked (Table 4) because induction had opposite effects on mite fitness in the two lines. However, the induction $\times$ selection line interaction was highly significant (Table 4).

\section{DISCUSSION}

Selection experiments are a powerful tool to investigate genetic trade-offs and the potential for adaptation. By selecting on particular traits, we have the ability to observe associations that may otherwise be difficult to detect using standard quantitative genetic techniques (Fry 1990). As in this selection experiment, local adaptation to host plants may be genetically cor-

TABLE 2. ANOVA table for costs of mite adaptation to a novel host plant.

\begin{tabular}{lrrrr}
\hline \hline \multicolumn{1}{c}{ Source of variation } & df & \multicolumn{1}{c}{ MS } & \multicolumn{1}{c}{$F$} & \multicolumn{1}{c}{$P$} \\
\hline Selection line & 1 & 7610.823 & 10.493 & 0.002 \\
Generation & 1 & 16448.781 & 22.678 & $<0.001$ \\
Selection line $\times$ Generation & 1 & 5820.329 & 8.024 & 0.006 \\
Error & 76 & 725.327 & & \\
\hline
\end{tabular}

Note: Fitness of mites in the c-line (control) and s-line (selection) was compared on favorable cotton plants at 5 and 20 mite generations (Fig. 3). 
TABLE 3. ANOVA table for the effects of cucurbitacins on spider mite fitness.

\begin{tabular}{lrrrc}
\hline \hline \multicolumn{1}{c}{ Source of variation } & df & \multicolumn{1}{c}{ MS } & \multicolumn{1}{c}{$F$} & \multicolumn{1}{c}{$P$} \\
\hline Cucurbitacins & 1 & 3615.391 & 5.662 & 0.019 \\
Selection line & 1 & 7134.776 & 11.173 & 0.001 \\
Cucurbitacins $\times$ Selection line $\dagger$ & 1 & 150.058 & 0.235 & 0.629 \\
Trial & 1 & 1635.353 & 2.561 & 0.112 \\
Error & 151 & 638.552 & & \\
\hline
\end{tabular}

Note: Fitness of c-line (control) and s-line (selection) mites was measured on favorable cotton plants painted with cucurbitacin-d in two trials.

$\dagger$ The other interaction terms with trial were not significant and are not shown.

related with reduced performance on other hosts and with altered host-plant preference. Adaptation to particular environments is often associated with responses in other phenotypic traits. If performance and preference are positively associated and vary quantitatively on alternative hosts, local disruptive selection may be able to maintain genetic variation in populations of herbivores. Trade-offs and adaptation to host plants
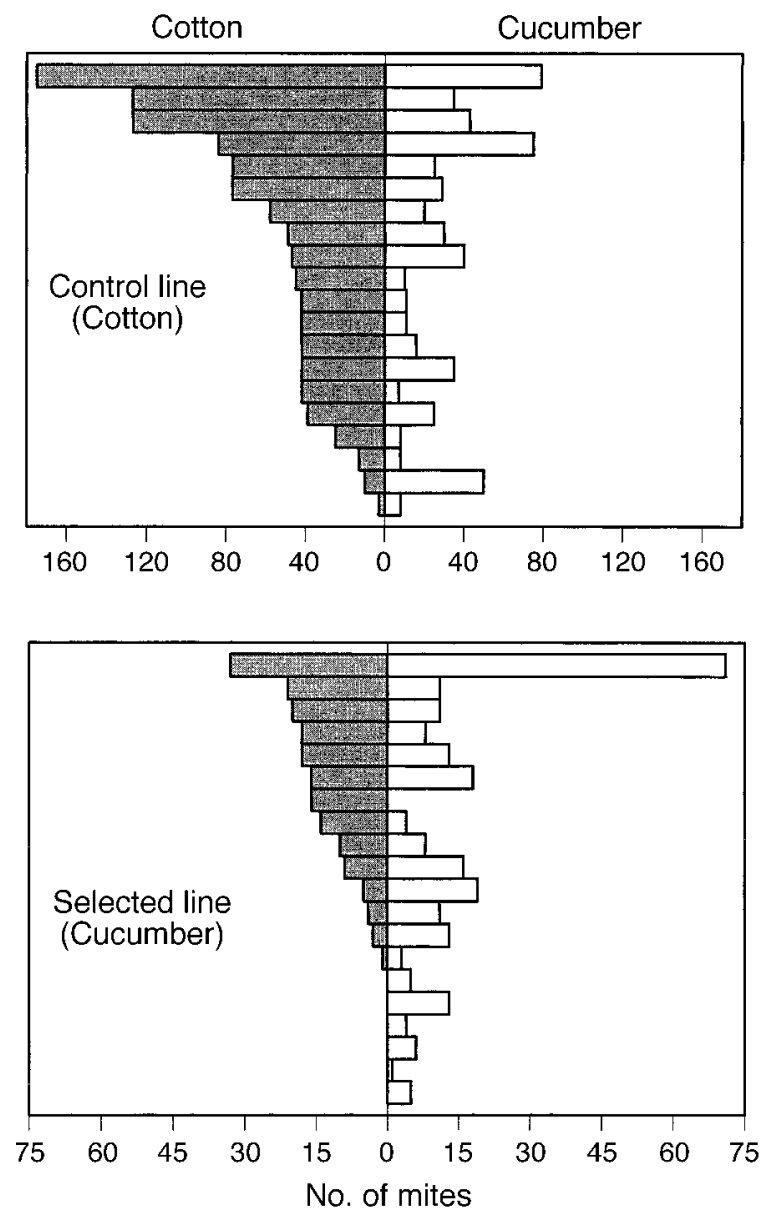

FIG. 5. The number of mites recovered from paired plants (one cotton and one cucumber). Mites were introduced from a $1-\mathrm{cm}^{2}$ square of bean leaf tissue infested with mites grown for $2 \mathrm{wk}$ on bean from either the control line (c-line) or the cucumber-adapted line (s-line). Each bar represents a single replicate and shows the numbers of mites found on each host. may often be subtle, and trade-offs may be difficult to detect by direct measurements, especially under apparently benign conditions.

In the current study, herbivorous spider mite populations established from recent field collections adapted to a poor-quality, novel host plant. These initial results mirror those of Gould (1979) and Fry (1990). My design controlled for potential maternal environmental effects when assaying the level of adaptation to the host plants and my results were unlikely to be caused by genetic drift, because mite populations were maintained in the thousands. Although spider mites adapted to a poor-quality host plant, a trade-off was not apparent when I measured fitness of the mites on the original favorable host. Fry (1992) suggested that testing for the trade-offs on such favorable host plants may offer no real challenge for the mites, and therefore may make detecting trade-offs in these situations unlikely. The costs of adaptation to cucumber were also not evident on zucchini, even though zucchini was overall a poor host, even for cucumber-adapted mites. Similarly, Gould (1979) and Fry (1992) found no evidence that costs of adaptation were more evident on other novel hosts. These repeatable results remain a puzzle, in that trade-offs in host use have not been detected as "costs" on alternative hosts, but only as the loss of adaptation in reverted populations.

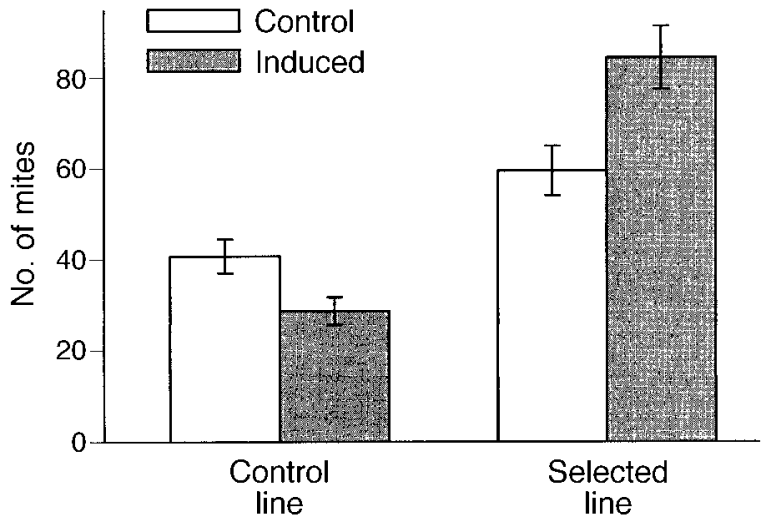

FIG. 6. Fitness of spider mites in the control line (c-line) and the cucumber-adapted line (s-line) on control cucumber plants and plants with induced resistance. Data are means \pm 1 SE. 
TABLE 4. ANOVA table for the effects of induced plant resistance on mites.

\begin{tabular}{lrrrc}
\hline \hline \multicolumn{1}{c}{ Source of variation } & df & \multicolumn{1}{c}{ MS } & \multicolumn{1}{c}{$F$} & \multicolumn{1}{c}{$P$} \\
\hline Induction & 1 & 795.733 & 0.871 & 0.353 \\
Selection line & 1 & 10117.341 & 11.081 & 0.001 \\
Trial & 1 & 5920.035 & 6.484 & 0.013 \\
Induction $\times$ Selection line $\dagger$ & 1 & 6815.107 & 7.464 & 0.008 \\
Error & 91 & 913.074 & & \\
\hline
\end{tabular}

Note: Fitness of mites from the c-line (control) and s-line (selection) was measured on induced and control cucumber plants (Fig. 6).

$\dagger$ This was the only relevant interaction term, indicating that the selection lines responded differently to the induction treatment.

A trade-off between performance on the two hostplant species was detected in this experiment, as in those of Gould and Fry. Reversion-line mites lost their high performance on the novel host to which they had recently become adapted within five generations. This loss of adaptation to cucumber in the r-line was probably due to relaxed selection pressure for high fitness on cucumber. Our results taken together indicate that trade-offs in host use of phytophagous mites appear to be a robust and repeatable phenomenon. Spider mites remain the best example of an extreme generalist that shows trade-offs in fitness on alternative host plants.

It is intriguing that mites from the reversion line not only lost their ability to have high fitness on cucumber, but they also had lower fitness than the control line. The reversion line of mites was started from several thousand individuals taken from the cucumber-selected line. It is possible that the process of selection increased the frequency of deleterious alleles closely linked to alleles for adaptation to cucumber (D. J. Futuyma, personal communication). It is possible then, that the apparent cost of adaptation to cucumber could be due to linkage, rather than a physiological trade-off. Unfortunately, performance of the reversion line was not assayed on favorable cotton plants.

Fitness of both lines of spider mites was low on zucchini plants (Fig. 4B). In addition, both lines were susceptible to cucurbitacin-d when painted on cotton leaves (Fig. 4A). This suggests that adaptation to the host-plant chemistry of cucumber was quite specific (i.e., to cucurbitacin-c), or that adaptation to cucumber was related to host-plant characters other than cucurbitacins. Cucurbitacin-c (cucumber) and -d (zucchini) are biochemically similar in structure, although they differ in the substitution at the C2, C3, C9 and C25 positions (Miro 1995). Adaptation to a novel host did not provide "cross resistance" or correlate with tolerance of closely related novel hosts. This finding contradicts one of the important assumptions of Ehrlich and Raven's (1964) classic proposal of herbivore and plant diversification, and questions the generality of adaptive zones. Adaptive-zone theory predicts that adaptation to a particular environment will be positively correlated with adaptation to similar environments. Even herbivores that are apparently adapted to particular host-plant families have been shown to be affected by subtle variations in host-plant chemistry (e.g., Huang and Renwick 1993). The complexity of intraspecific host-plant defenses may restrict host shifts to single species of novel host plants, and adaptive-zone shifts onto entire groups of plants may be rare.

Host-plant preference was significantly affected by my selection treatments. Control-line (c-line) mites reared on cotton showed a preference for cotton plants, even when reared on beans for at least one generation. However, mites adapted to cucumbers (the s-line) showed no preference for cotton or cucumber (Fig. 5). Given that c-line mite fitness was significantly higher on cotton plants than on cucumber, and s-line performance was high on both hosts, the results are consistent with the mites making adaptive host-plant choices.

Fry (1989) similarly found that spider mites adapted to tomato (Lycopersicon esculentum) had higher host "acceptance" of tomato than mites reared on relatively more favorable bean plants. Gotoh et al. (1993) also addressed host-plant preference in two strains of spider mites, one collected from tomatoes and the other from cucumber. In Y-tube olfactometer tests, tomato-strain mites showed a preference for tomato (over cucumber), while cucumber-strain mites showed no preference. However, in choice experiments using leaf discs, both strains of mites showed a clear preference for the host plant from which they had been collected. In addition, tomato-strain mites mated assortatively with other tomato-strain mites, although cucumber-strain mites did not show such patterns (Gotoh et al. 1993). These studies demonstrated that performance and preference of host-plant use may be genetically associated by linkage or pleiotropy, and evolve as correlated traits.

An alternative hypothesis is that the performancepreference correlation is due to the same trait determining preference and performance (D. J. Futuyma, personal communication). It is possible that adaptation to novel hosts in spider mites largely consists of a genetic loss of a deterrent response. Thus, mites adapted to new hosts are not deterred and thus increase feeding compared to "unadapted" mites. Unfortunately, the direct action and mechanisms of cucurbit phytochemistry on spider mites is unknown. Cucurbitacins per se do not affect mite preference (Gould 1978). Although no study has selected for host-plant preference to novel hosts and measured correlated changes in performance, 
this would be a fruitful avenue to study the evolution of diet specialization.

I have characterized induced resistance of cucumber plants to spider mite herbivory in detail elsewhere (Agrawal et al. 1999). Induction is associated with systemic increases in cucurbitacins. Initial damage to the cotyledons induced systemic resistance to c-line mites and decreased mite fitness on the first true leaf. The first true leaf was only a bud when the initial damage was applied. Surprisingly, in both trials conducted, the mites adapted to cucumber (s-line) had higher fitness on induced plants than on controls. This is counterintuitive because it appears that the mites have not only adapted to the resistance and associated induced cucurbitacins, but are somehow benefiting from the induction. It is possible that s-line mites evolved to be specialists on cucumber and use cucurbitacin-c, although it is more probable that the mites in the s-line colony adapted to some other property of induced plants. For example, mites are unlikely able to synthesize sterols, and thus may have derived limiting nutrients from steroidal cucurbitacins. S-line mites were maintained in a colony that was likely to be largely composed of induced plants with high levels of cucurbitacins.

\section{CONCLUSION}

These and previous results demonstrate that tradeoffs exist in host use by a spider mite. It appears that this adaptation is not associated with general tolerance or detoxification of cucurbitacins because it did not confer higher fitness for selected mites reared on a related species compared to unselected control-line mites. Adaptation to novel host plants may be acute. Adapted mites had higher fitness on induced plants than on undamaged control plants. Selection for high performance on a novel host resulted in correlated changes in host-plant preference, providing evidence that performance and preference are genetically correlated. Future selection experiments on both performance and preference components of novel host use will lead to greater insights into the causes and generality of tradeoffs and performance-preference correlations. Selection experiments combined with the study of naturally diverged populations may be the best way to detect the pleiotropic effects of adaptation to particular environments (e.g., Giamoustaris and Mithen 1995, Mithen et al. 1995). Such pleiotropic effects may explain the maintenance of variation of particular traits and why herbivores tend to be specialized.

\section{ACKNOWLEDGMENTS}

I thank Rick Karban, Douglas Tallamy, Jim Fry, and Fred Gould for providing encouragement and helpful advice. Dan Crocker (Peto Seeds, Saticoy, California) donated the $\mathrm{Cu}$ cumis sativus var. Marketmore 76 seeds. Chris Wardlaw maintained the mite colonies. The manuscript was improved by the comments of Rick Karban, Douglas Futuyma, Jennifer Thaler, Jay Rosenheim, Douglas Tallamy, Jim Campbell, Sharon Lawler, Lynn Adler, Rodrigo Mercader, and an anon- ymous reviewer. This study was funded by grants from the Center for Population Biology (U.C. Davis), Humanities Award (U.C. Davis), Jastro Shields Award (U.C. Davis), Northern California Chapter of Phi Beta Kappa, and National Science Foundation Dissertation Improvement Grant DEB9701109.

\section{Literature Cited}

Abrahamson, W. G., and A. E. Weis. 1997. Evolutionary ecology across three trophic levels. Princeton University Press, Princeton, New Jersey, USA.

Adler, L. S., J. Schmitt, and M. D. Bowers. 1995. Genetic variation in defensive chemistry in Plantago lanceolata (Plantaginaceae) and its effect on the specialist herbivore Junonia coenia (Nymphalidae). Oecologia 101:75-85.

Agrawal, A. A. 1998. Induced responses to herbivory and increase plant performance. Science 279:1201-1202.

Agrawal, A. A. 1999. Induced responses to herbivory in wild radish: effects on several herbivores and plant fitness. Ecology 80:1713-1723.

Agrawal, A. A., P. M. Gorski, and D. W. Tallamy 1999. Polymorphism in plant defense against herbivory: constitutive and induced resistance in Cucumis sativus. Journal of Chemical Ecology in press.

Agrawal, A. A., and R. Karban. 1997. Domatia mediete plant-arthropod mutualism. Nature 387:562-563.

Bernays, E., and M. Graham. 1988. On the evolution of host specificity in phytophagous arthropods. Ecology 69:886892.

Bi, J. L., J. B. Murphy, and G. W. Felton. 1997. Antinutritive and oxidative components as mechanisms of induced resistance in cotton to Helicoverpa zea. Journal of Chemical Ecology 23:97-117.

Björkman, C., S. Larsson, and R. Bommarco. 1997. Oviposition preferences in pine sawflies: a trade-off between larval growth and defence against natural enemies. Oikos 79:45-52.

Bowers, M. D. 1993. Aposematic caterpillars life-styles of the warningly colored and unpalatable. Pages 331-371 in N. E. Stamp And T. M. Casey, editors. Caterpillars: ecological and evolutionary constraints on foraging. Chapman \& Hall, New York, New York, USA.

Courtney, S. P., and T. T. Kibota. 1990. Mother doesn't know best: Selection of hosts by ovipositing insects. Pages 161188 in E. A. Bernays, editor. Insect-plant interactions II. CRC Press, Boca Raton, Florida, USA.

Da Costa, C. P., and C. M. Jones. 1971. Cucumber beetle resistance and mite susceptibility controlled by the bitter gene in Cucumis sativus L. Science 172:1145-1146.

Ehrlich, P. R., and P. H. Raven. 1964. Butterflies and plants: a study in coevolution. Evolution 18:586-608.

Fox, L. R., and P. A. Morrow. 1981. Specialization: Species property or local phenomenon? Science 211:887-893.

Fry, J. D. 1989. Evolutionary adaptation to host plants in a laboratory population of the phytophagous mite Tetranychus urticae Koch. Oecologia 81:559-565.

Fry, J. D. 1990. Trade-offs in fitness on different hosts: evidence from a selection experiment with a phytophagous mite. American Naturalist 136:569-580.

Fry, J. D. 1992. On the maintenance of genetic variation by disruptive selection among hosts in a phytophagous mite. Evolution 46:279-283.

Fry, J. D. 1996. The evolution of host specialization: Are trade-offs overrated? American Naturalist 148:S84-S107.

Futuyma, D. J., and G. Moreno. 1988. The evolution of ecological specialization. Annual Review of Ecology and Systematics 19:207-234.

Giamoustaris, A., and R. Mithen. 1995. The effect of modifying the glucosinolate content of leaves of oilseed rape (Brassica napus ssp. oleifera) on its interaction with spe- 
cialist and generalist pests. Annals of Applied Biology 126 347-363.

Gotoh, T., J. Bruin, M. W. Sabelis, and S. B. J. Menken. 1993. Host race formation in Tetranychus urticae: genetic differentiation, host plant preference and mate choice in a tomato and a cucumber strain. Entomologia Experimentalis et Applicata 68:171-178.

Gould, F. 1978. Resistance of cucumber varieties to Tetranychus urticae: genetic and environmental determinants. Journal of Economic Entomology 71:680-683.

Gould, F. 1979. Rapid host range evolution in a population of the phytophagous mite Tetranychus urticae Koch. Evolution 33:791-802.

Halaweish, F. T., and D. W. Tallamy. 1993. Quantitative determination of cucurbitacins by high performance liquid chromatography and high performance thin layer chromatography. Journal of Liquid Chromatography 16:497511.

Huang, X., and J. A. A. Renwick. 1993. Differential selection of host plants by two Pieris species: the role of oviposition stimulants and deterrents. Entomologia Experimentalis et Applicata 68:59-69

Jaenike, J. 1990. Host specialization in phytophagous insects. Annual Review of Ecology and Systematics 21:243273.

Janzen, D. H. 1985. A host plant is more than its chemistry. Illinois Natural History Survey Bulletin 33:141-174.

Jeppson, L. R., H. H. Keifer, and E. W. Baker. 1975. Mites injurious to economic plants. University of California Press, Berkeley, California, USA.

Joshi, A., and J. N. Thompson. 1995. Trade-offs and the evolution of host specialization. Evolutionary Ecology 9 82-92.

Karban, R. 1989. Fine-scale adaptation of herbivorous thrips to individual host plants. Nature 340:60-61.

Karowe, D. N. 1990. Predicting host range evolution: Colonization of Coronilla varia by Colias philodice (Lepidoptera: Pieridae). Evolution 44:1637-1647.

MacKenzie, A. 1996. A trade-off for host plant utilization in the black bean aphid, Aphis fabae. Evolution 50:155162.

Mayhew, P. J. 1997. Adaptive patterns of host-plant selection by phytophagous insects. Oikos 79:417-428.

Metcalf, R. L., and R. L. Lampman. 1989. The chemical ecology of Diabroticites and Cucurbitaceae. Experientia (Basel) 45:240-247.

Metcalf, R. L., R. A. Metcalf, and A. M. Rhodes. 1980.
Cucurbitacins as kairomones for diabroticite beetles. Proceedings of the National Academy of Sciences (USA) 77: 3769-2772.

Miro, M. 1995. Cucurbitacins and their pharmacological effects. Phytotherapy Research 9:159-168.

Mithen, R., A. F. Raybould, and A. Giamoustaris. 1995. Divergent selection for secondary metabolites between wild populations of Brassica oleracea and its implications for plant-herbivore interactions. Heredity 75:472-484.

Navajas, M. 1998. Host plant associations in the spider mite Tetranychus urticae (Acari: Tetranychidae): insights from molecular phylogeography. Experimental and Applied Acarology 22:201-214.

Price, P. W. 1994. Phylogenetic constraints, adaptive syndromes, and emergent properties: from individuals to population dynamics. Researches on Population Ecology 36: $3-14$.

Rosenthal, G. A., D. L. Dahlman, and D. H. Janzen. 1978. L-canaline detoxification: a seed predator's biochemical mechanism. Science 202:528-529.

Sadras, V. O., and L. J. Wilson. 1997. Growth analysis of cotton crops infested with spider mites. II. Partitioning of dry matter. Crop Science 37:492-497.

Schultz, J. C. 1988. Many factors influence the evolution of herbivore diets, but plant chemistry is central. Ecology 69: 896-897.

Tallamy, D. W., J. Stull, N. P. Ehresman, P. M. Gorski, and C. E. Manson. 1997. Cucurbitacins as feeding and oviposition deterrents to insects. Environmental Entomology 26:678-683.

Thompson, J. N. 1988. Evolutionary ecology of the relationship between oviposition preference and performance of offspring in phytophagous insects. Experimentalis et Applicata 47:3-14.

Thompson, J. N. 1994. The coevolutionary process. University of Chicago Press, Chicago, Illinois, USA.

Thompson, J. N. 1996. Trade-offs in larval performance on normal and novel hosts. Entomologia Experimentalis et Applicata 80:133-139.

Via, S. 1991. The genetic structure of host plant adaptation in a spatial patchwork: demographic variability among reciprocally transplanted pea aphid clones. Evolution 45: 827-852.

Wilson, L. T., C. H. Pickett, T. F. Leigh, and J. R. Carey. 1987. Spider mite (Acari: Tetranychidae) infestation foci: cotton yield reduction. Journal of Economic Entomology 16:614-617. 